\title{
Snail and Sonic Hedgehog activation in neuroendocrine tumors of the ileum
}

\author{
Volker Fendrich ${ }^{2,4}$, Jens Waldmann ${ }^{4}$, Farzad Esni ${ }^{2}$, Annette Ramaswamy ${ }^{5}$, \\ Michael Mullendore ${ }^{1,3}$, Malte Buchholz ${ }^{4}$, Anirban Maitra ${ }^{1,3}$ and \\ Georg Feldmann,
}

Departments of ${ }^{1}$ Pathology, ${ }^{2}$ Department of Surgery and ${ }^{3}$ The Sol Goldman Pancreatic Cancer Research Center, Johns Hopkins University School of Medicine, Baltimore, MD21231, USA

Departments of ${ }^{4}$ Surgery and ${ }^{5}$ Pathology, Philipps-Universität Marburg, Baldingerstraße, D-35043 Marburg, Germany

(Correspondence should be addressed to V Fendrich; Email: fendrich@med.uni-marburg.de)

\begin{abstract}
The transcription factor Snail represses E-cadherin and induces epithelial-mesenchymal transition, a process also exploited by invasive cancer cells. Aberrant Hedgehog $(\mathrm{Hh})$ signaling was recently observed in a variety of epithelial cancers and it has been shown that the Hh target gene Gli1 induces expression of Snail. In this study, we examined whether Snail and Sonic Hedgehog $(\mathrm{SHH})$ are expressed in neuroendocrine tumors (NETs) of the ileum. Using immunohistochemistry, we found expression of Snail in 22 out of $37(59 \%)$ of evaluated NET samples, but not in adjacent normal tissues. Snail expression was mostly restricted to the invasive front of the tumors. Six of seven liver metastases analyzed were positive for Snail. Intratumoral expression of SHH was detected in 27 out of $37(73 \%)$ tumors. As opposed to Snail, cells expressing SHH were found to be distributed more randomly throughout the tumors. Out of 30 primary NETs, $16(53 \%)$ showed both Snail and SHH expression. Furthermore, we found downregulation of E-cadherin in Snail-expressing cells by immunofluorescence. Real-time RT-PCR revealed conservation of the Hh target genes Gli1, Gli2, and Ptch in the pancreatic carcinoid cell line BON-1, which were downregulated upon Hh inhibition with cyclopamine. Moreover, $\mathrm{Hh}$ inhibition attenuated in vitro cell growth in a dose-dependent manner. In conclusion, we describe for the first time that Snail and $\mathrm{SHH}$ are overexpressed in a large subset of NETs of the ileum. Aberrant activation of these pathways might be involved in invasion and metastatic spread in NETs.
\end{abstract}

Endocrine-Related Cancer (2007) 14 865-874

\section{Introduction}

Neuroendocrine tumors (NETs) of the gastrointestinal (GI) tract are a poorly understood group of lesions that encompass a broad category of neoplasms derived from neuroendocrine cells of the GI mucosa. As a result of increasing incidence over the past 25 years, NETs are approximately as common as esophageal and gastric cancer, occurring in an estimated 38.5 per million in the US population (Maggard et al. 2004). In this heterogenous group, the 5-year survival rate lies around $60 \%$ for NETs of the ileum which comprise the majority of the tumors (Rorstad 2005). The presence of metastases, especially to the liver, is associated with a reduced 5-year survival.

The increased motility and invasiveness of cancer cells in the first phase of metastasis are reminiscent of epithelial-mesenchymal transition
(EMT) during embryonic development. In EMT, epithelial cells acquire fibroblast-like properties and show reduced intercellular adhesion and increased motility (Zhou \& Hung 2005). Numerous observations support the idea that EMT has a central role in tumor progression. During progression to metastatic competence, carcinoma cells acquire mesenchymal gene-expression patterns and properties. This results in changed adhesive properties and the activation of proteolysis and motility, which allows the tumor cells to metastasize and establish secondary tumors at distant sites (Thiery \& Sleeman 2006). During EMT, the E-cadherin promoter is frequently repressed by specific transcriptional repressors, including Snail, Slug, and Twist and by subsequent promoter hypermethylation. Of the transcriptional repressors, the 
best studied is Snail, a highly unstable protein. It is rapidly phosphorylated by glycogen synthase kinase-3 $\beta$ (GSK-3 $\beta$ ) and subsequently degraded by the ubiquitin-proteasome pathway. Conversely, inhibition of GSK-3 $\beta$ function results in upregulation of Snail by an NF- $\kappa \mathrm{B}-d e p e n d e n t$ pathway, loss of E-cadherin expression, and EMT. Additional protein modification further stabilizes Snail protein and promotes EMT and tumor invasion (Christofori 2006). Expression of Snail in epithelial tumors increases their aggressiveness, as seen in experimentally induced breast tumors, where high Snail expression correlates with an increased risk of tumor relapse and poor survival rates in human breast cancer (Moody et al. 2005).

Recently, aberrant activation of the Hedgehog (Hh) pathway has been found in the majority of upper aerodigestive tract epithelial cancers of endodermal lineage (Berman et al. 2003). In a recent study, Louro et al. (2002) showed that the Hh target gene Glil can rapidly and directly induce expression of Snail.

The purpose of the present study is to examine the in vivo expression of Snail and the Hh ligand Sonic Hedgehog (SHH) in NETs of the ileum and to clarify whether the Hh pathway is activated in BON-1 human carcinoid cells.

\section{Patients and methods}

\section{Subjects}

A series of 37 NETs of the ileum, including seven liver metastases, were obtained from the tissue bank of the Department of Pathology, Philipps-University of Marburg, Germany. Histological diagnoses were confirmed by an experienced pathologist (A R). Histopathological diagnosis and grading were done in accordance to Klöppel (2004). The immunohistochemistry results for Snaill and SHH were scored as follows: negative, $<5 \%$ of cells positive;,$+<30 \%$ of tumor cells positive; ++ , $>30 \%$ of tumor cells positive.

The study protocol conformed to the guidelines of the local ethics committee.

\section{Immunostaining}

For immunolabeling, formalin-fixed and paraffinembedded archived tumor samples and corresponding normal tissues were stained as previously described (Esni et al. 2004). Concentrations and sources of primary antibodies are listed in Table 1 . Briefly, slides from archived NETs were heated to $60^{\circ} \mathrm{C}$ for $1 \mathrm{~h}$, deparaffinized using xylene, and hydrated by a graded series of ethanol washes. Antigen retrieval was accomplished by microwave heating in $10 \mathrm{mM}$ sodium citrate buffer of $\mathrm{pH} 6.0$ for $10 \mathrm{~min}$. For immunohistochemistry, endogenous peroxidase activity was quenched by 10 -min incubation in $3 \% \mathrm{H}_{2} \mathrm{O}_{2}$. Nonspecific binding was blocked with $10 \%$ serum. Sections were then probed with primary antibodies overnight at $4{ }^{\circ} \mathrm{C}$. For immunohistochemistry, bound antibodies were detected using the avidin-biotin complex (ABC) peroxidase method (ABC Elite Kit, Vector Labs, Burlingame, CA, USA). Final staining was developed with the Sigma FAST DAB peroxidase substrate kit (Sigma). For immunofluorescence, bound antibodies were developed with the secondary antibody (Jackson Immuno Research, West Grove, PA, USA) and corresponding Cy3-, Cy2-, and Cy5conjugated antibodies (Jackson Immuno Research).

Mammary carcinoma samples from our tissue bank, which had previously been shown to express high levels of Snail, were used as positive controls along with each batch of Snail IHC stainings. SHH expression in crypts of adjacent normal ileum tissues, which has been described previously (van den Brink et al. 2002), was used as internal positive control for SHH immunohistochemistry.

\section{Fluorescence microscopy}

Fluorescence confocal microscopy was performed with a confocal laser scanning microscope (410LSM, Zeiss) using $40 \times($ NA 1.2) C-apochromat or $100 \times($ NA 1.4) objectives. The sections were scanned for Cy3-tagged (excitation $543 \mathrm{~nm}$ ) and FITC-tagged (excitation $488 \mathrm{~nm}$ ) markers. Sections (ten sections with images $512 \times 512$ pixels) were scanned at a pixel size of $0.07 \mathrm{~mm}$ and step size of $1 \mathrm{~mm}$. Image analysis was

Table 1 Working dilutions of antibodies used for immunohistochemistry and immunofluorescence

\begin{tabular}{llll}
\hline Antibody & Species & Working dilution & Source \\
\hline$\alpha$-E-cadherin & Rat & $1: 200$ & Zymed, San Francisco, CA, USA \\
$\alpha$-SHH & Goat & $1: 50$ & R\&D Sytems, Minneapolis, MN, USA \\
$\alpha-\beta$-Catenin & Rabbit & $1: 500$ & Santa Cruz Biotechnology, Santa Cruz, CA, USA \\
$\alpha$-Snail & Goat & $1: 100$ & Santa Cruz Biotechnology \\
Chromogranin A & Mouse & $1: 100$ & Santa Cruz Biotechnology \\
\hline
\end{tabular}


performed using MetaMorph series 5.0 software (Universal Imaging Corp). For specimens not requiring confocal analysis, images were obtained on an inverted motorized microscope (Axiovert 200M, Zeiss) outfitted with Cy5, Cy3, FITC, and DAPI filters. Threeand four-color images were captured using a Zeiss Axiocam, and pseudocolors assigned using Zeiss Axiovision software.

\section{Cell culture}

BON-1 pancreatic carcinoid cells (Parekh et al. 1994) were grown in DMEM/FK-12 (Invitrogen) supplemented with $10 \%$ fetal calf serum (FCS; Invitrogen) and $1 \times$ Pen/Strep (Biofluids, Camarillo, CA, USA) at $37^{\circ} \mathrm{C}$ in a humidified atmosphere containing $5 \% \mathrm{CO}_{2}$. The cell line was routinely tested for mycoplasma infection using the MycoSensor PCR Assay Kit (Stratagene, La Jolla, CA, USA).

\section{Proliferation assays}

Cells were seeded into 96-well plates at a density of 2000 cells in $200 \mu \mathrm{l}$ full medium/well and incubated overnight. Next, they were starved in low-serum medium $(0.5 \%$ FBS) for $24 \mathrm{~h}$. Then, medium was replaced for $100 \mu \mathrm{l}$ low-serum medium supplemented with vehicle, $1.5,3$, or $6 \mu \mathrm{M}$ cyclopamine, respectively, for 6 days. Untreated cells served as controls and wells containing medium only were used for background correction. To determine viable cell mass, $20 \mu \mathrm{l}$ of 3-(4,5-dimethylthiazolyl-2)2,5-diphenyltetrazolium bromide reagent (Promega) were added per well. Plates were incubated at $37^{\circ} \mathrm{C}$ for another $60 \mathrm{~min}$ and optical density read at $490 \mathrm{~nm}$ on a Wallac 1420 plate reader (Perkin-Elmer, Boston, MA, USA). All experiments were done in triplicate.

\section{RNA extraction and real-time quantitative RT-PCR}

Cells in the log growth phase were serum starved in $0.5 \%$ FCS for 1 day and then incubated with cyclopamine $(6 \mu \mathrm{M})$ or ethanol for another 4 days. Next, cells were lysed in the culture dish with $600 \mu \mathrm{l}$ buffer RLT and whole RNA extracted using the RNeasy kit (Qiagen) with on-column DNA digestion following the standard protocol provided by the manufacturer.

Human tissue samples were homogenized with a rotor stator homogenizer (Polytron PT1200C, Kinematica, Newark, NJ, USA) in $600 \mu$ lysis buffer RLT (Qiagen). Total RNA was then extracted using the RNeasy kit (Qiagen) following the standard protocol.

The mRNA was reverse transcribed into cDNA with oligo-dT primers using the Superscript First
Strand System for RT-PCR (Invitrogen) at $42{ }^{\circ} \mathrm{C}$ for 50 min. For amplification of cDNA specific for Ptch1, Gli1, Gli2, Gli3, SHH, and PGK-1 Assays-on-Demand (Applied Biosystems, Foster City, CA, USA) were used together with the QuantiTect Probe PCR kit (Qiagen). All PCRs were carried out on a 7300 Real-Time PCR System (Applied Biosystems) over 40 cycles, with denaturation for $15 \mathrm{~s}$ at $95^{\circ} \mathrm{C}$ and combined annealing/extension at $60{ }^{\circ} \mathrm{C}$ for $1 \mathrm{~min}$. Determination of Snail mRNA was performed over 40 cycles with $15 \mathrm{~s}$ of denaturation at $95^{\circ} \mathrm{C}$, annealing at $61{ }^{\circ} \mathrm{C}$ for $20 \mathrm{~s}$, extension at $72{ }^{\circ} \mathrm{C}$ for $30 \mathrm{~s}$ and data acquisition at $78{ }^{\circ} \mathrm{C}$ using the Quantitect SYBR Green PCR kit (Qiagen); primer sequences were forward: $5^{\prime}$-ggttcttctgcgctactgct- $3^{\prime}$ and reverse: $5^{\prime}$-tagggetgctggaaggtaaa- $3^{\prime}$. Relative fold mRNA expression levels were determined using the $2\left(-\Delta \Delta C_{\mathrm{t}}\right)$ method (Livak \& Schmittgen 2001) with PGK1 (phosphoglycerate kinase 1) as housekeeping control.

\section{Statistical analysis}

Kruskal-Wallis analysis was performed using SPSS version 14.0 for Microsoft Windows. Two-tailed $t$-test was performed using GraphPad Prism for Windows version 4.0. $P<0.05$ was regarded as statistically significant. Prism was also used to generate boxplots in Figs $1 \mathrm{E}$ and 2E.

\section{Results \\ Patients}

Eighteen males and nineteen females with a median age of 63 years (range 34-91 years) at the time of surgery were included in this study. All NETs were histologically well-differentiated and tested positive for chromogranin A by IHC (not shown). Clinical characteristics are listed in Table 2.

\section{Overexpression of Snail in NETs of the ileum}

Immunohistochemical staining revealed expression of Snail in 22 out of $37(59 \%)$ of NETs tested (Table 2). In tumors, in which Snail was found to be expressed, Snailpositive cells were not distributed homogeneously throughout the whole tumor. Instead, Snail expression was mostly restricted to peripheral areas rendering what appeared to be the invasive front of the tumors, i.e., where malignant cells invade bordering non-malignant tissue (Fig. 1A and B). Fifteen tumors did not show any Snailpositive cells (1C). Six of seven (86\%) liver metastases analyzed were positive for Snail in IHC (Fig. 1D). In normal intestinal epithelium, Snail staining was absent in 

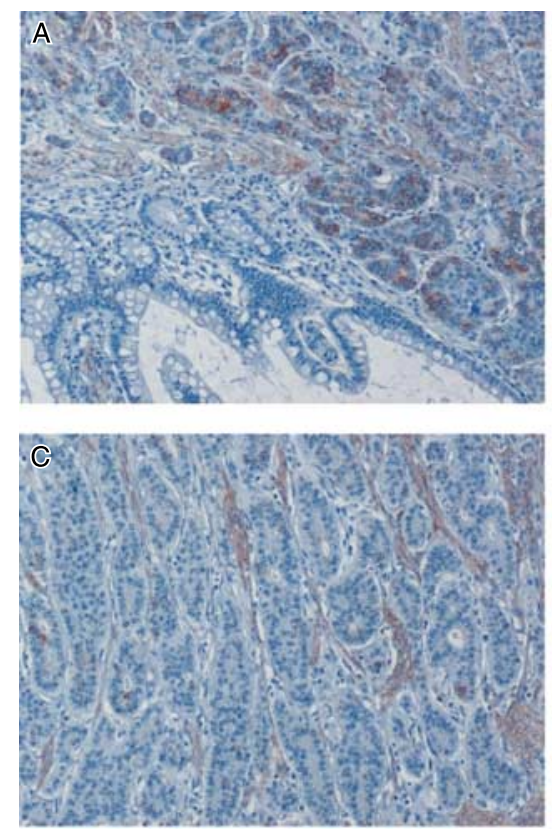
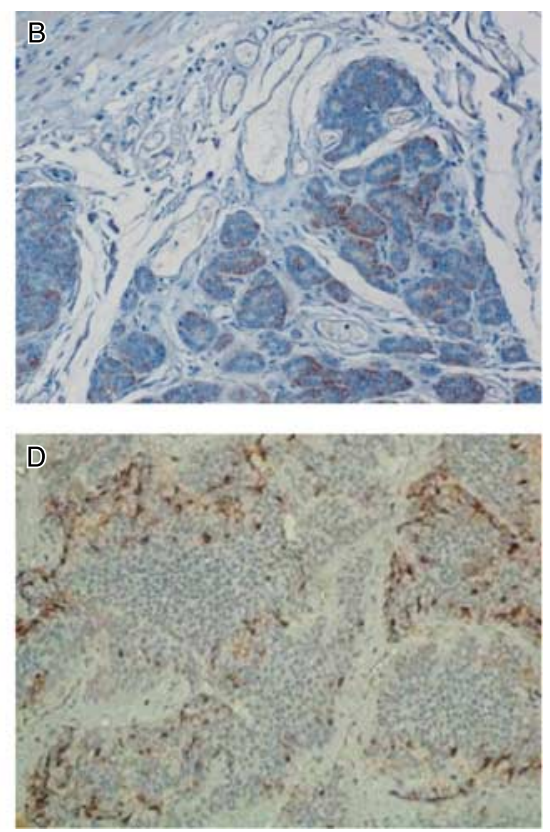

$\mathrm{E}$

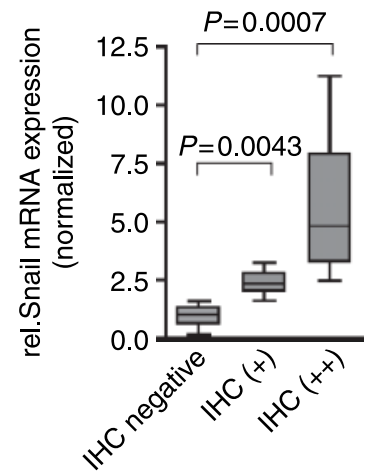

Figure $1 \mathrm{IHC}$ staining for Snail in tissue samples from human GI-NETs. (A) Representative example of Snail expressing carcinoid and adjacent normal tissue lacking Snail expression (lower half of image). (B) In Snail-positive samples, Snail was not expressed homogenously throughout the whole tumor, but was mostly limited to the invasive front. (C) In some of the examined carcinoid samples, no Snail expression could be found in the tumor, but only in mesenchymal cells. (D) All liver metastases showed Snail expression. (E) Steady-state Snail mRNA levels were determined by real-time RT-PCR in a subset of 19 samples. In each group, the upper and lower hinges of the boxes mark the 25th and 75th percentiles respectively; whiskers denote the ranges.

all samples. Correlation of intratumoral Snail overexpression with clinicopathological features was not analyzed due to the small sample number.

These results were confirmed at the mRNA level on a subset of 19 samples using quantitative real-time RT-PCR. We found increased steady-state Snail mRNA levels in samples that were classified as ' + ' $(P=0.0043)$ or ' $++'(P=0.0007)$ at the protein level, respectively, when compared with samples with negative IHC staining (Fig. 1E).

\section{Overexpression of SHH}

Intratumoral expression of the Hh ligand $\mathrm{SHH}$ was detected in 27 out of 37 (73\%) tumors studied (Table 2). As opposed to Snail, cells expressing SHH were found to be distributed more randomly throughout larger areas of the tumors (Fig. 2A). A total of ten tumors did not show any SHH-positive cells (Fig. 2C). Three of the seven (43\%) liver metastases also expressed SHH (Fig. 2D). As expected, $\mathrm{SHH}$ expression was also seen in normal intestinal epithelium (Fig. 2B).

SHH mRNA levels tended to be higher in samples that were classified as positive by IHC, but these differences did not reach statistical significance, possibly due to the small sample number and considerable variation between different cases, as well as SHH expression in krypts of non-malignant, normal intestine (Fig. 2E).

Out of 30 primary NETs, 16 (53\%) showed both Snail and SHH expressions, whereas six (20\%) were negative for both markers. Eight (27\%) primary NETs which expressed SHH showed no Snail staining, but none of the primaries expressed Snail in the absence of SHH.

Three of six liver metastases, which expressed Snail, lacked expression of SHH as seen in IHC (Table 2). Of four pairs of primary tumors with matched liver metastasis tissue samples from the same patients included in this series, Snail expression was found in all four metastases, but only in two of four primaries. In case number 30 , the primary tumor was classified as ' + ' for snail by IHC, whereas the matched metastasis is ' ++ '.

Neither Snail nor SHH protein expression was detected in non-malignant liver tissue adjacent to metastatic foci.

\section{Suppression of E-cadherin expression by Snail in NETs}

To examine the influence of Snail on E-cadherin expression in NETs, immunofluorescence analysis for 

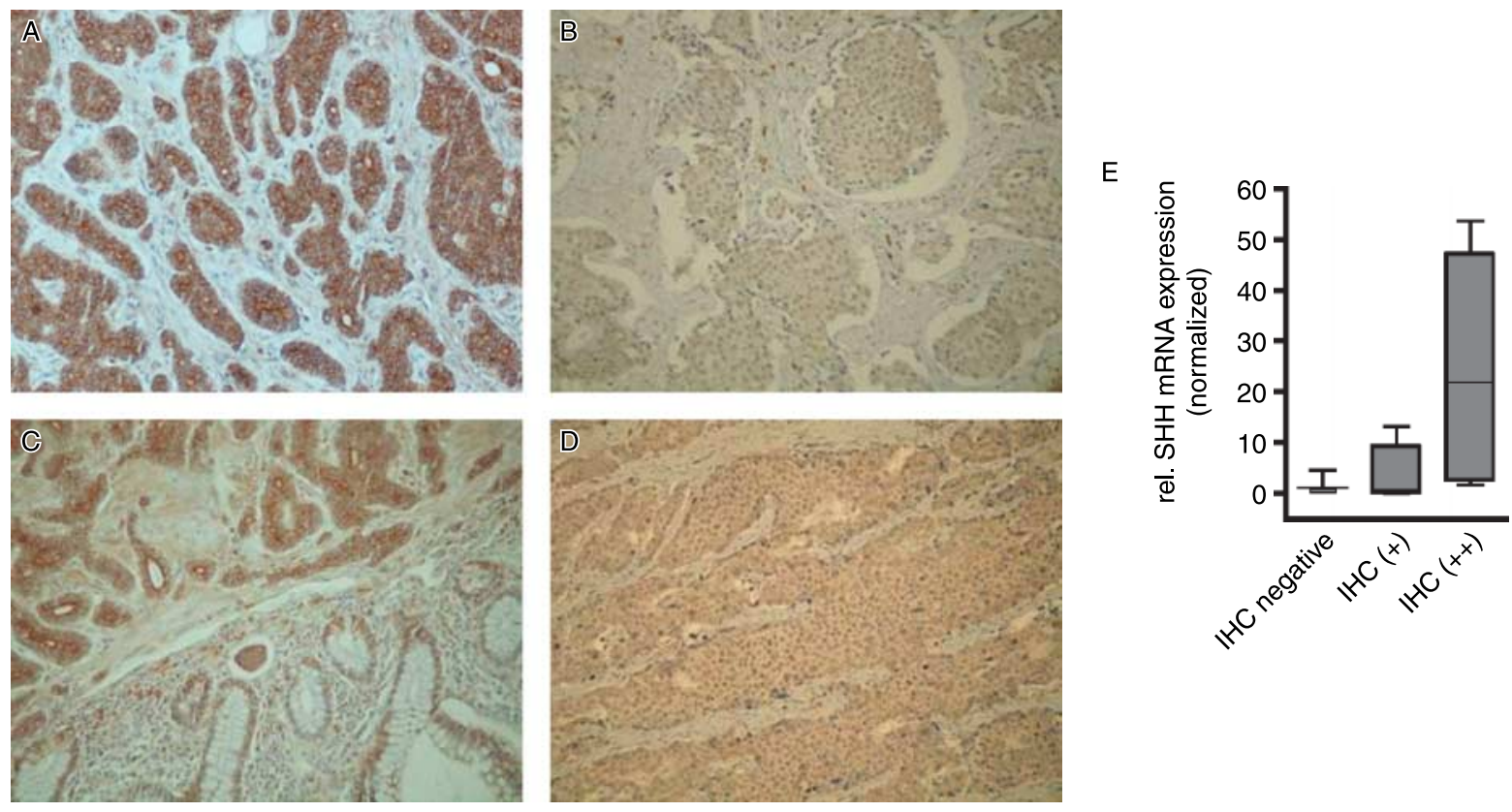

Figure $2 \mathrm{IHC}$ staining for SHH in tissue samples from human GI-NETs. Marked overexpression of SHH was seen in the majority of studied samples (A), whereas some completely lacked expression of SHH (B). SHH expression was also detected in normal intestinal epithelium (C). Two of four liver metastases also expressed SHH (D). (E) Steady-state SHH mRNA levels as revealed by real-time RT-PCR. Results are presented as boxplots as described above.

Snail, E-cadherin, and $\beta$-catenin was performed in a subset of eight randomly selected Snail-positive NETs of the ileum (Fig. 3). E-cadherin was found to be downregulated on Snail-expressing cells, while high levels of E-cadherin were expressed abundantly on the surfaces of cells lacking Snail expression (Fig. 3A and C). $\beta$-Catenin was expressed on the membranes of all cells irrespective of presence or absence of Snail protein and was used as control counterstaining in areas with suppressed E-cadherin expression (Fig. 3B).

\section{Conservation of Hh pathway in BON-1 cells}

The pancreatic carcinoid cell line BON-1 was used for in vitro studies. Quantitative real-time RT-PCR analysis revealed expression of the Hh pathway-related genes Glil, Gli2, and Ptch. Upon Hh inhibition with the small molecule-smoothened antagonist cyclopamine, all of these target genes were found to be downregulated, in line with preexisting aberrant activation of the $\mathrm{Hh}$ pathway in this cell line. Interestingly, expression of the inhibitory transcription factor Gli3 was not altered upon Hh inhibition (Fig. 4A).

Furthermore, we found that $\mathrm{Hh}$ inhibition with cyclopamine reduced BON1 cell growth in vitro in a dose-dependent manner (Fig. 4B), further supporting our hypothesis of aberrant $\mathrm{Hh}$ activation in this tumor entity.

\section{Discussion}

The GI tract is the predominant site of origin for NETs, which were formerly called carcinoids, although they can occur throughout the body. NETs are generally slow growing but frequently metastasize to the liver, ranking second to colorectal carcinoma as a source of isolated liver metastases (Chen et al. 1998). Molecular data on NETs of the GI tract have been accumulating in recent years, but the genetic basis of endocrine tumor development and progression is still poorly understood.

EMT occurs during embryonic morphogenesis in multicellular organisms, in which embryonic mesenchymal cells are formed and become motile following the loss of epithelial cell polarity. In recent years, EMT has also been recognized as a potential mechanism for cancer progression (Thiery 2002). Cancer cells undergoing EMT have lost specific target recognition and are usually equipped with autocrine loops of growth signals, mechanisms to evade apoptosis, and the potential to elicit angiogenesis for independent nutrient supply (Gotzmann et al. 2004). A central event in EMT is downregulation of E-cadherin that leads to the loss of cell-cell contact and the consecutive progression of the cells toward a malignant phenotype. The transcription factor Snail is one major suppressor of E-cadherin and a strong inducer of EMT. Snail downregulates 
Table 2 Clinical characteristics and results of Snail and Sonic Hedgehog (SHH) immunohistochemistry in 33 patients with neuroendocrine tumors of the ileum

\begin{tabular}{|c|c|c|c|c|c|c|c|c|c|}
\hline Patient no. & Age (Years) & Sex & NET localization & Histology & $\mathbf{K i}-67(\%)^{\mathrm{a}}$ & LN Mtx ${ }^{\mathrm{b}}$ & Liver Mtx $^{\mathrm{C}}$ & Snail1 expression & SHH expression \\
\hline 1 & 57 & $M$ & Liver metastasis & & ND & ND & ND & Negative & Negative \\
\hline 2 & 57 & $\mathrm{~F}$ & Ileum carcinoid & Well-differentiated & ND & ND & ND & ++ & ++ \\
\hline 3 & 73 & $M$ & Liver metastasis & & ND & ND & ND & ++ & ++ \\
\hline 4 & 61 & M & lleum carcinoid & Well-differentiated & ND & + & + & Negative & Negative \\
\hline 5 & 77 & $\mathrm{~F}$ & Ileum carcinoid & Well-differentiated & ND & ND & ND & ++ & ++ \\
\hline 6 & 56 & $M$ & Ileum carcinoid & Well-differentiated & 30 & + & + & + & + \\
\hline 7 & 72 & $M$ & Ileum carcinoid & Well-differentiated & 3 & + & + & ++ & ++ \\
\hline 8 & 52 & $M$ & Ileum carcinoid & Well-differentiated & 1 & + & - & ++ & ++ \\
\hline 9 & 63 & $\mathrm{~F}$ & Ileum carcinoid & Well-differentiated & 20 & + & + & + & ++ \\
\hline 10 & 42 & $M$ & Ileum carcinoid & Well-differentiated & 1 & + & + & Negative & + \\
\hline 11 & 68 & $M$ & Ileum carcinoid & Well-differentiated & 1 & + & - & ++ & + \\
\hline 12 & 91 & $M$ & Ileum carcinoid & Well-differentiated & 1 & + & + & Negative & + \\
\hline 13 & 60 & $\mathrm{~F}$ & Ileum carcinoid & Well-differentiated & 5 & + & + & ++ & + \\
\hline 14 & 55 & $\mathrm{~F}$ & Ileum carcinoid & Well-differentiated & 5 & + & + & + & ++ \\
\hline 15 & 52 & $\mathrm{~F}$ & Ileum carcinoid & Well-differentiated & 1 & + & + & Negative & + \\
\hline 16 & 66 & $\mathrm{~F}$ & Liver metastasis & & 1 & + & + & + & Negative \\
\hline 17 & 57 & $M$ & lleum carcinoid & Well-differentiated & 5 & + & - & Negative & ++ \\
\hline 18 & 66 & $M$ & Ileum carcinoid & Well-differentiated & 3 & + & + & + & + \\
\hline 19 & 67 & $\mathrm{~F}$ & Ileum carcinoid & Well-differentiated & 1 & + & + & Negative & Negative \\
\hline \multirow[t]{2}{*}{20} & 66 & $\mathrm{~F}$ & lleum carcinoid & Well-differentiated & 2 & + & + & Negative & ++ \\
\hline & & & Liver metastasis & & ND & & & + & Negative \\
\hline 21 & 62 & M & Ileum carcinoid & Well-differentiated & 17 & + & + & Negative & Negative \\
\hline 22 & 44 & $M$ & Ileum carcinoid & Well-differentiated & 5 & + & + & Negative & Negative \\
\hline \multirow[t]{2}{*}{23} & 43 & $\mathrm{~F}$ & Ileum carcinoid & Well-differentiated & 5 & + & + & + & + \\
\hline & & & Liver metastasis & & ND & & & + & + \\
\hline 24 & 39 & $M$ & lleum carcinoid & Well-differentiated & 2 & + & + & ++ & ++ \\
\hline 25 & 79 & $M$ & Ileum carcinoid & Well-differentiated & 1 & + & - & + & ++ \\
\hline 26 & 67 & $\mathrm{~F}$ & Ileum carcinoid & Well-differentiated & 5 & + & - & Negative & ++ \\
\hline 27 & 70 & $M$ & Ileum carcinoid & Well-differentiated & 1 & + & - & ++ & ++ \\
\hline 28 & 65 & $\mathrm{~F}$ & Ileum carcinoid & Well-differentiated & 1 & + & + & + & ++ \\
\hline 29 & 74 & $F$ & Ileum carcinoid & Well-differentiated & ND & ND & ND & Negative & ++ \\
\hline \multirow[t]{2}{*}{30} & 40 & $M$ & Ileum carcinoid & Well-differentiated & 4 & + & + & + & + \\
\hline & & & Liver metastasis & & ND & & & ++ & Negative \\
\hline 31 & 75 & $\mathrm{~F}$ & Ileum carcinoid & Well-differentiated & 1 & + & - & Negative & Negative \\
\hline 32 & 91 & M & Ileum carcinoid & Well-differentiated & 10 & + & + & Negative & Negative \\
\hline \multirow[t]{2}{*}{33} & 34 & $\mathrm{~F}$ & Ileum carcinoid & Well-differentiated & 1 & + & + & Negative & + \\
\hline & & & Liver metastasis & & ND & & & + & + \\
\hline
\end{tabular}

ND, no data available.

${ }^{\mathrm{a} K i}$-67 proliferation index in percent.

${ }^{b}$ Presence $(+)$ or absence $(-)$ of lymph node metastases at time of diagnosis.

cPresence $(+)$ or absence $(-)$ of liver metastases at time of diagnosis. 

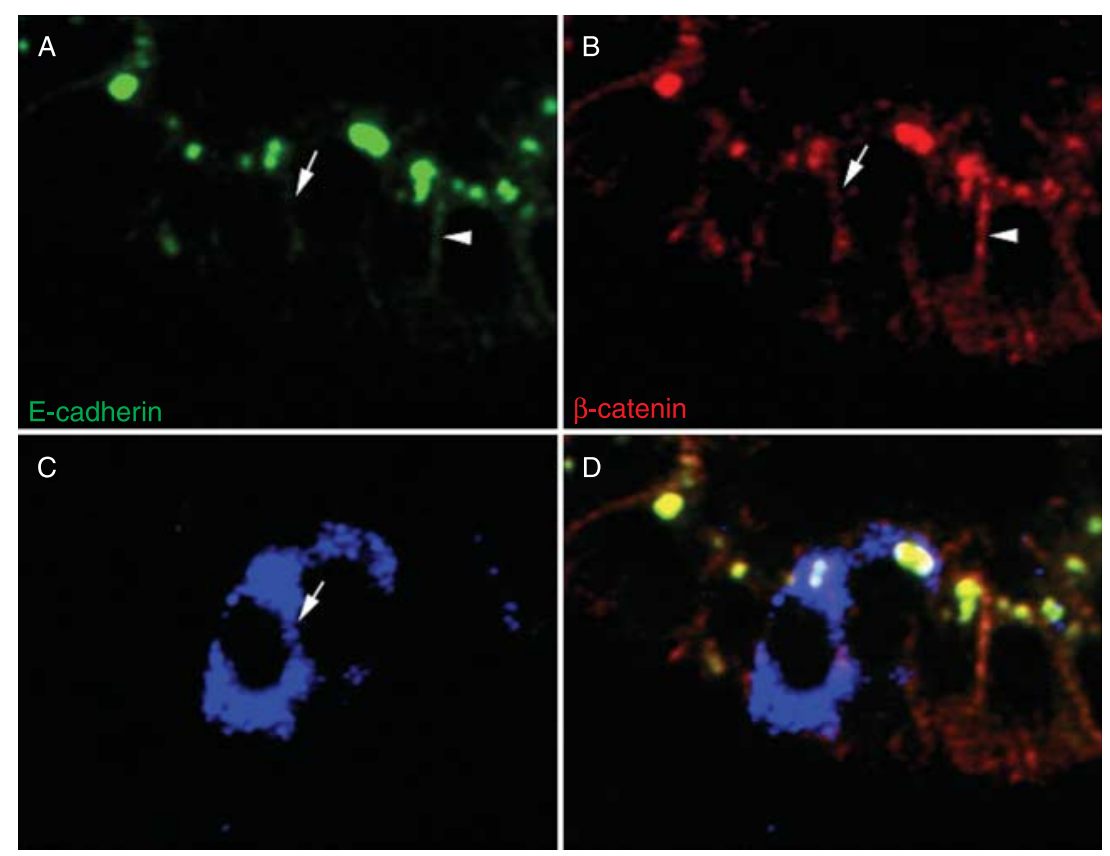

Figure 3 Immunofluorescence staining for (A) E-cadherin, (B) $\beta$-catenin, (C) Snail, and (D) merge. E-cadherin expression was downregulated on cells, in which Snail was expressed (arrow), whereas E-cadherin expression was seen on the surface of cells that did not stain for Snail (arrowhead). $\beta$-Catenin was expressed on surfaces of both Snail-positive and -negative cells. The figure shows results from one representative of eight performed experiments.

E-cadherin in different types of tumors, e.g., hepatocellular carcinomas (Jiao et al. 2002), carcinomas from the esophagus, cardia, stomach (Rosivatz et al. 2006), and colorectal carcinomas (Roy et al. 2005).

Our study is the first to show that Snail is expressed in NETs of the ileum, and to the best of our knowledge this is also the first report of Snail overexpression in an endocrine related cancer at all. We found significant expression of Snail in more than 50\% of the NETs we investigated. Our results are in line with a metaanalysis from Zikusoka et al. (2005) of comparative genomic hybridization data, which found that in up to $50 \%$ of all carcinoids there is a gain on chromosome 20q13, the location of Snail.

As seen in tumors induced in the skin of mice (Cano et al. 2000), Snail-expressing cells were mostly found at the invasive front of the NETs. At this site, tumor cells migrate into and invade the surrounding tissue either as single cells or in collective clusters. Figure 1 shows the Snail-positive invasive front of a NET of the ileum, with no Snail staining detectable in normal intestinal epithelium. The presence of hepatic metastases in GI NETs has obvious implications in terms of patients' quality of life and overall prognosis. The 5 -year survival rate in patients with hepatic metastases from midgut carcinoids is $0-50 \%$ as opposed to $75-99 \%$ in patients without liver metastases (Rorstad
2005). Six of seven (86\%) liver metastases analyzed in our study were positive for Snail, possibly supporting our hypothesis that Snail might be involved in the development of metastases by promoting a more invasive phenotype through EMT.

Our observation of lack of SHH and Snail expression in non-malignant normal liver tissue is in line with previous reports by others (Sugimachi et al. 2003, Sicklick et al. 2006), although the latter group detected Snail mRNA transcripts in adult hepatocytes by in situ hybridization.

To verify that in GI-NETs expression of Snail is associated with loss of E-cadherin, we performed immunofluorescence analysis for Snail, E-cadherin, and $\beta$-catenin. In fact, E-cadherin was found to be downregulated on Snail-expressing cells, while high levels of E-cadherin were expressed abundantly on the surfaces of cells lacking Snail expression (Fig. 3), a finding commonly related to Snail-induced EMT and metastasis (Huber et al. 2005).

Genes of the Hh pathway, commonly involved in embryonic patterning and stem cell maintenance in adult tissues, are frequently mutated in basal cell carcinomas (Athar et al. 2006) and medulloblastomas (Taylor et al. 2002). Moreover, recent studies by our own group as well as by others described aberrant activation of $\mathrm{Hh}$ signaling in several malignancies of 

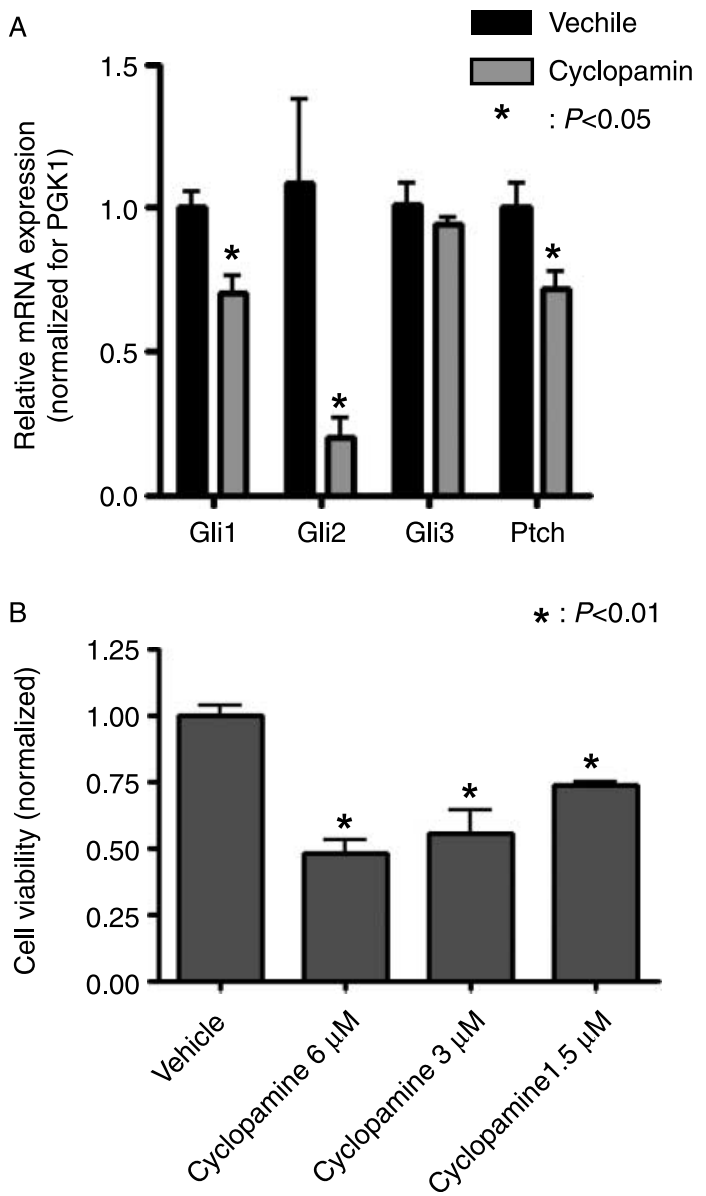

Figure 4 (A) Expression of Gli1, Gli2, Gli3, and Ptch mRNA in $\mathrm{BON}-1$ cells treated with solvent only or cyclopamine. Upon treatment with cyclopamine, RT-PCR revealed downregulation of all $\mathrm{Hh}$ target genes tested. (B) $\mathrm{Hh}$ inhibition with cyclopamine lead to reduced proliferation of $\mathrm{BON}-1$ cells in a dosedependent manner.

the aerodigestive tract (Berman et al. 2003, Thayer et al. 2003), and particularly high Hh pathway activity has been found in metastatic lesions of prostate and pancreatic cancers (Karhadkar et al. 2004, Feldmann et al. 2007), suggesting that Hh signaling might play a role in metastatic tumor spread. Exogenous expression of the Hh-dependent transcription factor Gli1 in lowmetastatic rodent prostate carcinoma cells caused enhanced migrationin vitro and development of visceral metastases in vivo, correlating with induction of Snail expression and E-cadherin repression. All these effects were completely inhibited by treatment with the pathway inhibitor cyclopamine, showing that the Hh pathway indeed contributes to EMT (Karhadkar et al. 2004). Recently, Li et al. (2006) showed in an elegant study that Snail is rapidly induced by Gli1 in vitro and in skin tumors. In line with these results, we found expression of SHH and Snail in $53 \%$ of primary NETs. Eight primary NETs expressed SHH without consecutive Snail expression, but no primary tumor expressed Snail in the absence of SHH. These results suggest that also in ileum NETs Snail is induced as a downstream target of SHH or, likewise, Gli1. Overall, we found expression of SHH in 27 out of $37(73 \%)$ ileum-derived NET samples analyzed (Table 2). SHH-positive cells were distributed throughout larger areas of the tumors. As expected, SHH expression was also seen in normal intestinal epithelium (Nielsen et al. 2004).

Our in vivo findings are in line with the in vitro expression of Hh-related genes Gli1, Gli2, Gli3, and Ptch in BON-1 cells (Fig. 4). After treating this cell line with cyclopamine, we found a significant downregulation of the Hh target genes Glil, Gli2, and Ptch at the mRNA level, as demonstrated by RT-PCR (Fig. 4). Furthermore, we found that Hh inhibition with cyclopamine reduced cell growth in vitro in a dosedependent manner (Fig. 4). Although to date cyclopamine cannot be chemically synthesized but has to be extracted from the corn lily Veratrum californicum (Cooper et al. 1998) and is thus costly and difficult to obtain in larger amounts for clinical use, considerable research effort is currently undertaken to pharmacologically target the Hh-signaling pathway using new small molecule inhibitors, and our results suggest that the Hh pathway might constitute a potential therapeutic target in NETs. One such synthetic drug has recently been shown to reduce medulloblastoma growth in a transgenic mouse model (Romer et al. 2004).

In conclusion, our data presented here show that the sister pathways Snail and $\mathrm{Hh}$ are activated in a considerable subset of GI-NETs, leading to downregulation of E-cadherin in line with EMT. Hh activation is observed in BON-1 cells and Hh inhibition with cyclopamine reduces NET cell growth in vitro.

\section{Acknowledgements}

Cyclopamine was donated by Infinity Pharmaceuticals (Cambridge, MA, USA). Many thanks to Savita Bisht for checking the manuscript and to Malte Buchholz for performing real-time PCRs. This study was supported by the NCI grant R01CA113669 to A M. G F was supported by a fellowship grant within the Postdoc-Program of the German Academic Exchange Service (DAAD). V F was supported by a Research Grant of the University Medical Center Giessen and Marburg. The authors declare that there is no conflict of interest that would prejudice the impartiality of this scientific work. 


\section{References}

Apelqvist A, Ahlgren U \& Edlund H 1997 Sonic Hedgehog directs specialised mesoderm differentiation in the intestine and pancreas. Current Biology 7 801-804.

Athar M, Tanq X, Lee JL, Kopelovich L \& Kim AL 2006 Hedgehog signalling in skin development and cancer. Experimental Dermatology 15 667-677.

Berman DM, Karhadkar SS, Maitra A et al. 2003 Widespread requirement for hedgehog ligand stimulation in growth of digestive tract tumours. Nature 425 846-851.

Cano A, Pérez-Moreno MA, Rodrigo I, Locascio A, Blanco MJ, del Barrio MG, Portillo F \& Nieto MA 2000 The transcription factor snail controls epithelial-mesenchymal transitions by repressing Ecadherin expression. Nature Cell Biology 2 76-83.

Chen H, Hardacre JM, Uzar A, Cameron JL \& Choti MA 1998 Isolated liver metastases from neuroendocrine tumors: does resection prolong survival? Journal of the American College of Surgeons 187 88-92.

Christofori G 2006 New signals from the invasive front. Nature 44 444-450.

Cooper MK, Porter JA, Young KE \& Beachy PA 1998 Teratogen-mediated inhibition of target tissue response to SHH signaling. Science 280 1603-1607.

Devereux TR, Anna CH, Foley JF, White CM, Sills RC \& Barrett JC 1999 Mutation of beta-catenin is an early event in chemically induced mouse hepatocellular carcinogenesis. Oncogene 18 4726-4733.

Esni F, Stoffers DA, Takeuchi T \& Leach SD 2004 Origin of exocrine pancreatic cells from nestin-positive precursors in developing mouse pancreas. Mechanism of Development 121 15-25.

Feldmann G, Dhara S, Fendrich V, Bedja D, Beaty R, Mullendore M, Karikari C, Alvarez H, Iacobuzio-Donahue C, Jimeno A et al. 2007 Blockade of hedgehog signaling inhibits pancreatic cancer invasion and metastases: a new paradigm for combination therapy in solid cancers. Cancer Research 67 2187-2196.

Gotzmann J, Mikula M, Eger A, Schulte-Hermann R, Foisner R, Beug H \& Mikulits W 2004 Molecular aspects of epithelial cell plasticity: implications for local tumor invasion and metastasis. Mutation Research 566 9-20.

Huber MA, Kraut N \& Beuq H 2005 Molecular requirements for epithelial-mesenchymal transition during tumor progression. Current Opinion in Cell Biology 17 548-558.

Jiao W, Miyazaki K \& Kitajima Y 2002 Inverse correlation between E-cadherin and Snail expression in hepatocellular carcinoma cell lines in vitro and in vivo. British Journal of Cancer 86 98-101.

Karhadkar SS, Bova GS, Abdallah N, Dhara S, Gardner D, Maitra A, Isaacs JT, Berman DM \& Beachy PA 2004 Hedgehog signalling in prostate regeneration, neoplasia and metastasis. Nature 431 707-712.

Klöppel G, Perren A \& Heitz PU 2004 The gastroenteropancreatic neuroendocrine cell system and its tumors: the WHO classification. Annals of New York Academy of Sciences 1014 13-27.
Li X, Deng W, Nail CD, Bailey SK, Kraus MH, Ruppert JM \& Lobo-Ruppert SM 2006 Snail induction is an early response to Gli1 that determines the efficiency of epithelial transformation. Oncogene 25 609-621.

Livak KJ \& Schmittgen TD: 2001 Analysis of relative gene expression data using real-time quantitative PCR and the 2(-DeltaDelta $\mathrm{C}(\mathrm{T}))$ method. Methods 25 402-408.

Louro ID, Bailey EC, Li X, South LS, McKie-Bell PR, Yoder BK, Huang CC, Johnson MR, Hill AE, Johnson $\mathrm{RL}$ et al. 2002 Comparative gene expression profile analysis of Gli1 and c-Myc in an epithelial model of malignant transformation. Cancer Research 62 5867-5873.

Maggard MA, O'Connell JB \& Ko CY 2004 Updated population-based review of carcinoid tumors. Annals of Surgery 240 117-122.

Moody SE, Perez D, Pan TC, Sarkisian CJ, Portocarrero CP, Sterner CJ, Notorfrancesco KL, Cardiff RD \& Chodosh LA 2005 The transcriptional repressor Snail promotes mammary tumor recurrence. Cancer Cell 8 197-209.

Nielsen CM, Williams J, van den Brink GR, Lauwers GY \& Roberts DJ 2004 Hh pathway expression in human gut tissues and in inflammatory gut diseases. Laboratory Investigation 84 1631-1642.

Parekh D, Ishizuka J, Townsend CM Jr, Haber B, Beauchamp RD, Karp G, Kim SW, Rajaraman S, Greeley G Jr \& Thompson JC 1994 Characterization of a human pancreatic carcinoid in vitro: morphology, amine and peptide storage, and secretion. Pancreas 9 83-90.

Perl AK, Wilgenbus P, Dahl U, Semb H \& Christofori G 1998 A causal role for E-cadherin in the transition from adenoma to carcinoma. Nature 392 190-193.

Romer JT, Kimura H, Magdaleno S et al. 2004 Suppression of the $\mathrm{SHH}$ pathway using a small molecule inhibitor eliminates medulloblastoma in $\operatorname{Ptc1}(+/-)$ p53 $(-/-)$ mice. Cancer Cell 6 229-240.

Rorstad O 2005 Prognostic indicators for carcinoid neuroendocrine tumors of the gastrointestinal tract. Journal of Surgical Oncology 89 151-160.

Rosivatz E, Becker KF, Kremmer E, Schott C, Blechschmidt K, Hofler H \& Sarbia M 2006 Expression and nuclear localization of Snail, an E-cadherin repressor, in adenocarcinomas of the upper gastrointestinal tract. Virchows Archiv 448 277-287.

Roy HK, Smyrk TC, Koetsier J, Victor TA \& Wali RK 2005 The transcriptional repressor Snail is overexpressed in human colon cancer. Digestive Diseases and Sciences 50 42-46.

Sicklick JK, Li Y-X, Jayaraman A, Kannangai R, Qi Y, Vivekanandan P, Ludlow JW, Owzar K, Chen W, Torbenson MS et al. 2006 Dysregulation of the Hedgehog pathway in human hepatocarcinogenesis. Carcinogenesis 27 748-757. 
Sugimachi K, Tanaka S, Kameyama T, Taguchi K, Aishima S, Shimada M, Sugimachi K \& Tsuneyoshi M 2003 Transcriptional repressor Snail and Progression of Human Hepatocellular Carcinoma. Clinical Cancer Research 9 2657-2664.

Taylor MD, Liu L, Raffel C, Hui CC, Mainprize TG, Zhang X, Agatep R, Chiappa S, Gao L, Lowrance A et al. 2002 Mutations in SUFU predispose to medulloblastoma. Nature Genetics 31 306-310.

Thayer SP, di Magliano MP, Heiser PW, Nielsen CM, Roberts DJ, Lauwers GY, Qi YP, Gysin S, Fernandez-del Castillo C, Yajnik V et al. 2003 Hedgehog is an early and late mediator of pancreatic cancer tumorigenesis. Nature 425 851-856.

Thiery JP 2002 Epithelial-mesenchymal transitions in tumour progression. Nature Review Cancer 2 442-454.

Thiery JP \& Sleeman JP 2006 Complex networks orchestrate epithelial-mesenchymal transitions. Nature Review. Molecular and Cellular Biology 7 131-142. van den Brink GR, Hardwick JC, Tytgat GN, Brink MA, Ten Kate FJ, Van Deventer SJ \& Peppelenbosch MP 2001 Sonic Hedgehog regulates gastric gland morphogenesis in man and mouse. Gastroenterology 121 317-328.

van den Brink GR, Hardwick JCH, Nielsen C, Xu C, ten Kate FJ, Glickman J, van Deventer SJ, Roberts DJ \& Peppelenbosch MP 2002 Sonic Hedgehog expression correlates with fundic gland differentiation in the adult gastrointestinal tract. Gut 51 628-633.

Watkins DN, Berman DM, Burkholder SG, Wang B, Beachy PA \& Baylin SB 2003 Hedgehog signalling within airway epithelial progenitors and in small-cell lung cancer. Nature 422 313-317.

Zhou BP \& Hung MC 2005 Wnt, hedgehog and snail: sister pathways that control by GSK-3beta and beta-Trcp in the regulation of metastasis. Cell Cycle 4 772-776.

Zikusoka MN, Kidd M, Eick G, Latich I \& Modlin IM 2005 The molecular genetics of gastroenteropancreatic neuroendocrine tumors. Cancer 104 2292-2309. 